\title{
Multifocal Pupillography Identifies Ranibizumab-Induced Changes in Retinal Function for Exudative Age-Related Macular Degeneration
}

\author{
Faran Sabeti, ${ }^{1}$ Ted Maddess, ${ }^{1}$ Rohan W. Essex, ${ }^{1,2}$ and Andrew C. James ${ }^{1}$
}

Purpose. To investigate the efficacy of multifocal pupillographic objective perimetry (mfPOP) to quantify the effects of intravitreal ranibizumab injection for choroidal neovascularization (CNV) secondary to exudative age-related macular degeneration (AMD).

Methods. mfPOP visual fields from 20 patients with unilateral exudative AMD treated with intravitreal ranibizumab were measured before and after 3 months of treatment and were compared with that in 30 normal subjects. Two stimulus types consisting of ensembles of 24 or 44 independent stimuli per eye had a mean presentation interval at each region of 1 second. Pupil responses were recorded with video cameras under infrared illumination. Multiple linear models were fitted to contraction amplitudes and delay to peak responses, to determine the independent effects of exudative AMD before and after ranibizumab therapy.

RESUlts. After 3 months of treatment, mean additional response delays compared to normal subjects for the 24-region stimulus improved significantly $\left(P<5 \times 10^{-9}\right)$ from a mean of $18.82 \pm 3.0 \mathrm{~ms}$ at baseline to $7.45 \pm 3.15 \mathrm{~ms}$. The mean effect of exudative AMD at baseline decreased constriction amplitude by $-1.11 \pm 0.24 \mathrm{~dB}(P<0.00001)$ with little improvement after ranibizumab therapy. Small pretreatment elevations of extrafoveal sensitivity correlated with improvements in central retinal thickness (CRT) after treatment $(P<$ $0.0005)$.

Conclusions. Improvements in mfPOP contraction amplitudes and time to peak responses were measured in eyes treated with intravitreal ranibizumab; however, response delays appeared to be the most indicative of functional improvement. Confirmation of hypersensitivity in the extrafoveal field in a larger group may support this finding as a prognostic marker for good treatment outcomes. (Invest Ophthalmol Vis Sci. 2012;53: 253-260) DOI:10.1167/iovs.11-8004

From the ${ }^{1}$ ARC Centre of Excellence in Vision Science, The John Curtin School of Medical Research, The Australian National University, Canberra, Australian Capital Territory (ACT), Australia; and the ${ }^{2} \mathrm{Oph}-$ thalmology Department, The Australian National University, Canberra Hospital, ACT, Australia.

Supported by the Australian Research Council (ARC) through the ARC Centre of Excellence in Vision Science (CE0561903), AusIndustry, and Seeing Machines, Ltd., Canberra. The sponsor or funding organization had no role in the design or conduct of this research.

Submitted for publication June 6, 2011; revised October 12 and November 24, 2011; accepted November 28, 2011.

Disclosure: F. Sabeti, None; T. Maddess, Seeing Machines (C, F), P; R.W. Essex, None; A.C. James, Seeing Machines (C, F), P

Corresponding author: Faran Sabeti, ARC Centre of Excellence in Vision Science, The John Curtin School of Medical Research, ANU, Canberra ACT 0200, Australia; faran.sabeti@anu.edu.au.
Choroidal neovascularization (CNV) secondary to age-related macular degeneration (AMD) is the leading cause of severe vision loss in adults in the Western world. ${ }^{1}$ The trigger for new blood vessel growth is associated with the release of vascular endothelial growth factor (VEGF). ${ }^{2,3}$ The subsequent proliferation of $\mathrm{CNV}$ begins the destructive changes in retinal morphology and initiates the symptoms associated with exudative AMD. Recent pharmaceutical therapies have therefore focused on inhibiting VEGF expression. One such anti-VEGF therapy, ranibizumab (Lucentis; Novartis, North Ryde, NSW, Australia) has been shown to cause regression of CNV. ${ }^{4,5}$

Traditionally, indications for treatment, and retreatment have been determined by measuring best corrected visual acuity (BCVA) and changes in retinal structures with optical coherence tomography (OCT). However, good correlation between morphologic change after ranibizumab therapy and BCVA is not always present. ${ }^{6}$ Particularly, early recurrence of disease after treatment often does not affect BCVA, suggesting that visual acuity may not be the best measure of visual function. Alternative means of evaluating retinal sensitivity may identify disease activity earlier or identify which subjects will benefit from treatment.

Central retinal sensitivity, as measured by perimetry, has been shown to improve significantly with ranibizumab treatment alone $e^{6,7}$ and in combination with photodynamic therapy. ${ }^{8}$ Field loss identified by multifocal pupillographic objective perimetry (mfPOP) has been shown to correlate highly with perimetric visual field loss. ${ }^{9,10}$ Our laboratory has combined $\mathrm{mfPOP}$ and so-called spatially sparse stimuli from mfVEPs $^{11,12}$ to deliver high diagnostic accuracy in glaucoma and early-stage diabetic retinopathy. ${ }^{13,14}$ More recently, we have shown that higher density mfPOP stimulus arrays tailored for assessing the macula are feasible, ${ }^{15}$ and these have provided excellent diagnostic accuracy in early-stage AMD (Sabeti F, et al. IOVS 2010;51:ARVO E-Abstract 2794).

In this study, we examined the effects of intravitreal ranibizumab therapy on visual function assessed by the new macular mfPOP stimuli in patients with CNV secondary to AMD. In particular, we evaluated changes in regional sensitivity and response delay before and after 3 months of treatment and whether these can predict if treatment will be effective.

\section{MeTHODS}

\section{Patient Selection}

This study included 20 patients with unilateral exudative AMD recruited from the ophthalmology department at The Canberra Hospital (Table 1). The affected eyes included 13 with occult and 7 with predominantly classic choroidal neovascularization $(\mathrm{CNV})$. There were 14 women and 6 men, mean age $( \pm \mathrm{SD}) 78.8 \pm 7.2$ years. The subjects were assessed for their suitability for ranibizumab injections after BCVA, fundus biomicroscopy, fluorescein angiography, and OCT (Cirrus: Carl Zeiss Meditec, Inc., Dublin, CA). Those subjects initially 
TABLE 1. Summary of Baseline Patient Data

Fundus Features

\begin{tabular}{|c|c|c|c|c|c|c|c|c|c|}
\hline \multirow[b]{3}{*}{ ID } & \multirow[b]{3}{*}{ Study Eye } & \multirow[b]{3}{*}{ Age $(y)$} & \multirow[b]{3}{*}{ Sex } & \multirow[b]{3}{*}{ BCVA } & \multirow[b]{3}{*}{ Diagnosis } & \multirow{2}{*}{\multicolumn{2}{|c|}{ Drusen }} & & \\
\hline & & & & & & & & \multirow[b]{2}{*}{ Hemorrhage Size $\ddagger$} & \multirow[b]{2}{*}{ Geographic Atrophy Size } \\
\hline & & & & & & Number* & Size† & & \\
\hline 401 & OD & 78 & M & 0.3 & Occult & 2 & 3 & - & - \\
\hline 402 & OS & 85 & $\mathrm{~F}$ & 0.3 & Occult & 1 & 3 & - & - \\
\hline 403 & OS & 73 & F & 1.0 & Classic & 2 & 2 & - & 3 \\
\hline 404 & OD & 68 & $\mathrm{~F}$ & 0.5 & Classic & 1 & 1 & 2 & - \\
\hline 405 & OD & 86 & F & $\delta$ & Occult & - & - & 5 & - \\
\hline 406 & OD & 72 & $\mathrm{~F}$ & 1.3 & Occult & 3 & 3 & 5 & - \\
\hline 407 & OD & 79 & M & 0.5 & Classic & - & - & - & 5 \\
\hline 408 & OS & 81 & $\mathrm{~F}$ & 0.8 & Occult & 1 & 3 & 2 & - \\
\hline 409 & OD & 78 & F & 0.3 & Occult & 2 & 3 & - & - \\
\hline 410 & OD & 79 & $\mathrm{~F}$ & 0.8 & Classic & 1 & 3 & - & - \\
\hline 411 & OD & 72 & M & $\int$ & Classic & 1 & 3 & - & - \\
\hline 412 & OD & 79 & M & 1.0 & Classic & 1 & 2 & - & - \\
\hline 413 & OD & 76 & M & 0.2 & Occult & 3 & 2 & - & 3 \\
\hline 414 & OS & 63 & $\mathrm{~F}$ & 0.6 & Occult & 3 & 3 & 5 & - \\
\hline 415 & OS & 85 & F & 0.3 & Occult & 2 & 3 & - & 5 \\
\hline 416 & OS & 89 & F & 0.5 & Occult & 3 & 3 & 1 & - \\
\hline 417 & OS & 80 & $\mathrm{~F}$ & 0.1 & Occult & 2 & 3 & 2 & - \\
\hline 418 & OS & 83 & M & 0.5 & Classic & 2 & 3 & - & - \\
\hline 419 & OS & 92 & $\mathrm{~F}$ & 0.3 & Occult & 3 & 3 & - & - \\
\hline 420 & OS & 79 & $\mathrm{~F}$ & 0.5 & Occult & 2 & 3 & - & - \\
\hline
\end{tabular}

*Drusen number: $1,<20 ; 2,>20 ; 3,>50$.

† Drusen size: $1,<63 \mu \mathrm{m} ; 2, \geq 63$ to $<125 \mu \mathrm{m} ; 3, \geq 125 \mu \mathrm{m}$.

$\ddagger$ Lesion size: $1,<500 \mu \mathrm{m} ; 2, \geq 500$ to $<1000 \mu \mathrm{m} ; 3, \geq 1000$ to $<1500 \mu \mathrm{m} ; 4, \geq 1500$ to $<2000 \mu \mathrm{m} ; 5,>3000 \mu \mathrm{m}$.

BCVA, count fingers.

received monthly intravitreal injections of $0.5 \mathrm{mg}$ ranibizumab for 3 months. Patients were excluded from the study if they had co-existing ocular or systemic pathology affecting visual function. Thirty control subjects ( 11 men, 19 women; mean age, $71.0 \pm 6.0$ years) were recruited and given a thorough eye examination involving detailed history, frequency-doubling perimetry C-20 field tests, slit lamp examination of the anterior segment, fundus biomicroscopy and applanation tonometry. The normal controls were required to have BCVA of $6 / 6$ or better and no history of ocular surgery or disease. The study was conducted according to the tenets of the Declaration of Helsinki. All subjects gave informed written consent, and the study adhered to the requirements of the Australian National University's Human Experimentation Ethics Committee (protocol 2010/194).

\section{Multifocal Pupillography Stimuli and Recording}

In the present study, all subjects were tested with two randomized stimulus protocols using a prototype version of an FDA-approved field analyzer (TrueField Analyzer; Seeing Machines Ltd., Acton, ACT, Australia). Testing occurred at baseline, on the same day as the first injection before dilation, and after three consecutive months of treatment. Our experimental design was chosen because earlier studies have demonstrated stabilization of BCVA from 3 months. ${ }^{5,16}$ We have described the mfPOP technique in detail elsewhere. ${ }^{13-15,17,18}$ The following is a brief account of the stimulus properties used in this study. The yellow multifocal stimuli were presented to each eye independently (dichoptic stimulation) on two LCD displays at optical infinity. This technique allows for afferent and efferent defects to be measured at all visual field locations. ${ }^{14}$ The stimulus ensemble consisted of a somewhat overlapping 44-region (O44) and a nonoverlapping 24-region (T24) dartboard layout (Fig. 1), extending from fixation to $\pm 15^{\circ}$ eccentricity. Details of these macular stimuli are given elsewhere. ${ }^{15}$ Large, somewhat overlapping stimuli, have been proposed to reduce test-retest variability. ${ }^{19}$ All stimuli were pseudorandomly presented at a mean interval of $1 / \mathrm{second} /$ region and transiently $(33 \mathrm{~ms})$ pulsed at a luminance of $250 \mathrm{~cd} / \mathrm{m}^{2}$. The stimuli were projected on a dim starburst background with a low spatial frequency radial grating pattern at a luminance of $10 \mathrm{~cd} / \mathrm{m}^{2}$ and included a small central fixation cross. The radial pattern and a long, thin, vertical, white line bisecting the fixation point were displayed to aid fusion. The subjects' gaze position was monitored during testing. Each test consisted of eight segments of 30 seconds' duration, with short rest breaks between segments to allow for blinking and recovery. Separate video cameras for each eye recorded pupil responses under infrared illumination. Pupil responses were measured by fitting a circle to the lower three fourths of the pupil margin providing some tolerance to ptosis.

\section{Data Analysis}

Recordings were exported from the field analyzer to a commercial software program (MatLab R2009b; The MathWorks, Natick, MA) for analysis. Linear regression was used to estimate the response amplitude and time to peak from the raw pupillary responses. ${ }^{20}$ Multiple linear regression is expressed as $-y=X \beta+\mathrm{e}$, where $y$ is the observed response, $X$ is the explanatory variable (independent variable), $\beta$ are the regression coefficients, and $e$ represents the errors in the noise. We fitted a log-normal function to the pupil response waveforms to analyze the following parameters: contraction amplitudes (in micrometers) and time to peak (in milliseconds). ${ }^{13,14,21}$ We used a previously described procedure to overcome the effects of miotic pupils by normalizing the responses to the mean pupil size and subsequently scaling them. ${ }^{14,17}$ The regressive method used to estimate mean pupil responses for each region generated standard errors for the measured parameters, ${ }^{14,17}$ and provided a tolerance of $15 \%$ data loss from blinks and fixation losses before a segment needed to be repeated.

Independent effects of treatment and subject variants on pupillary responses were measured by fitting a multiple linear model to the response amplitudes. Initially, the pupil response amplitudes were transformed to decibels to stabilize the variance as follows:

$$
\mathrm{Amp}_{\mathrm{dB}}=10 \log _{10} \mathrm{Amp}_{\mu \mathrm{m}}
$$

Time data were converted from seconds to milliseconds to aid interpretation and did not require transformation. The effects of inde- 


\section{Region O44 Layout Overlapping Polar Stimulus}
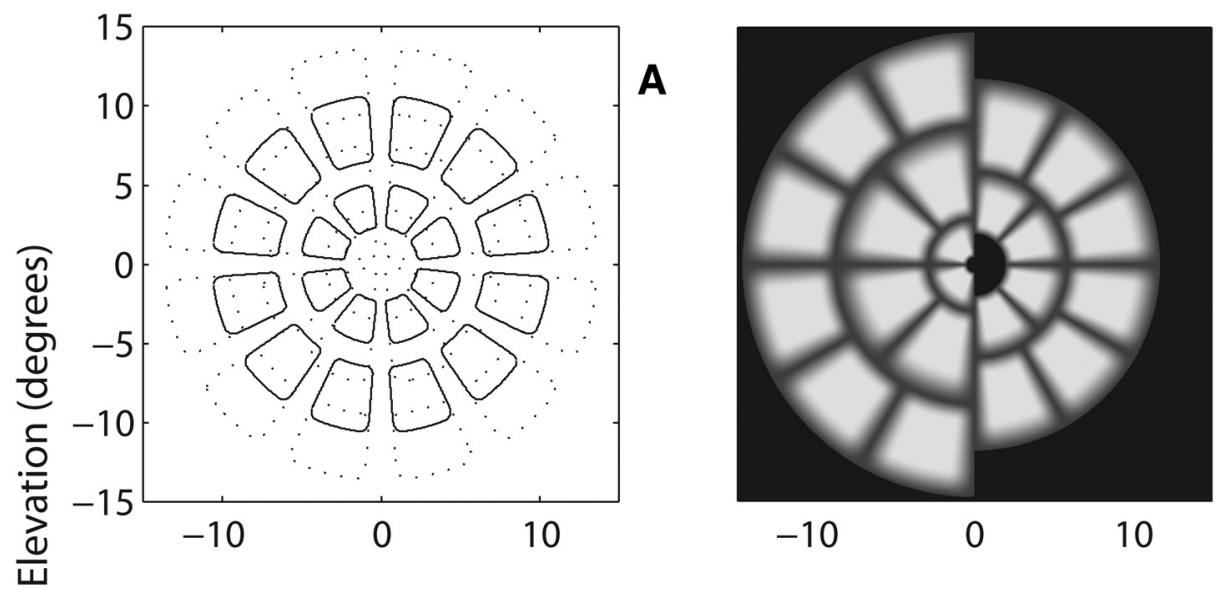

FigURE 1. Multifocal stimulus pattern arranged in a dartboard layout presented with all regions activated. (A) The 44-region layout consisted of five rings with overlapping polar stimuli (actual stimuli are yellow). (B) Half the stimuli from each hemifield are shown. (C, D) The 24-region layout arranged in three non-overlapping rings (labeled 1 to 3 ) with yellow stimuli. No stimuli infringed on the horizontal or vertical midlines.

\section{Region T24 Layout}

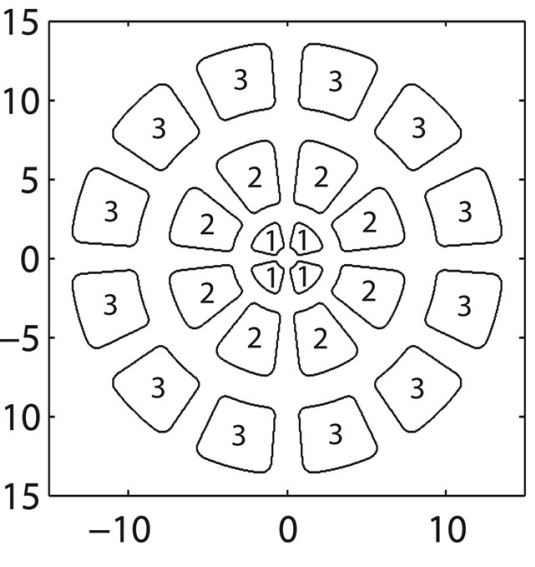

C

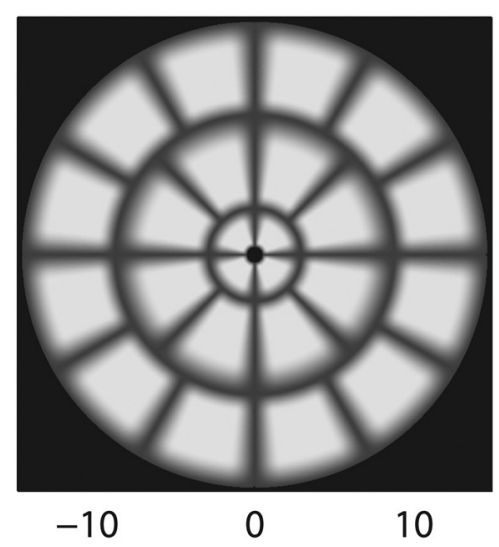

Visual Field Azimuth (degrees)

pendent factors were measured by fitting them as contrasts to the reference condition. The reference condition represented the mean direct pupillary response (computed across eyes, pupils, and visual field regions) of normal male subjects. This definition permitted the significance of contrasting effects, such as being female, AMD, and consensual responses to be calculated. To determine whether changes in retinal function were observed more in the parafoveal than in the foveal area, we compared the baseline and month 3 follow-up mfPOP responses in three concentric rings (Fig. 1C) for the T24 stimulus: (1) fovea, (2) parafovea, (3) perifovea (radii of $0.5^{\circ}-3.0^{\circ}, 3.3^{\circ}-7.7^{\circ}$, and $8.0^{\circ}-15^{\circ}$, respectively).

We used the leave-one-out method for estimating the area under the curve (AUC) of receiver operator characteristic (ROC) plots to determine the diagnostic status of subjects at pre- and posttreatment examinations and the diagnostic performance of our stimulus protocols, as previously described. ${ }^{13,14,17,21}$ The method used in this study calculated ROC AUC values from the between-eye (asymmetric) regional deviations relative to normal for both contraction amplitude and time to peak responses from normative data, a method that has also shown utility in mfVEPs. ${ }^{22}$

\section{RESULTS}

The mean pupillary contraction waveforms recorded at baseline and after three consecutive months of intravitreal ranibizumab injections from an 80-year-old woman are shown in Figure 2. Each region of the stimulus ensemble was independently modulated for each eye, producing separate direct and consensual responses concurrently. Regional response amplitudes in Figure 2A were found to be significantly reduced in the nasal hemisphere of the left eye. Figure 2B shows an improvement in mean contraction amplitudes at 3 months after treatment. Her logMAR BCVA improved from 1 to 0.18 , and mean central retinal thickness (CRT) decreased from 340 to $258 \mu \mathrm{m}$ at 3 months of follow-up (cf. Figs. $2 \mathrm{E}$ and $2 \mathrm{~F}$ ).

After the follow-up period the visual acuities improved in 12 of 20 patients, with eight eyes measuring no improvement. The mean baseline visual acuity value was $0.68 \pm 0.45$ (mean \pm SD) before treatment. After treatment, mean visual acuities did not improve significantly from baseline $(0.63 \pm 0.44, P=0.48)$. Mean central retinal thickness decreased significantly after three ranibizumab injections $(P<0.001)$ from $340 \pm 83 \mu \mathrm{m}$ at pretreatment to $236 \pm 36 \mu \mathrm{m}$ after treatment.

A multiple linear model was fitted to contraction amplitudes (in decibels, Table 2) and times to peak (Table 3) responses to determine the independent effect of AMD before and after ranibizumab injections and of stimulus variables on pupillary responses. Table 2 summarizes the reference condition, as described in the Methods section, and the effects of the subject's sex and exudative AMD at baseline and after three intravitreal ranibizumab injections for contraction amplitudes corrected for age. The average direct pupil contraction in normal male subjects (reference) ranged from $4.91 \pm 0.30 \mathrm{~dB}$ (mean \pm $\mathrm{SE}$ ) for stimulus $\mathrm{O} 44$ to $3.94 \pm 0.30 \mathrm{~dB}$ for $\mathrm{T} 24$. Before treatment, exudative AMD reduced mean response amplitudes by between $-0.71 \pm 0.17 \mathrm{~dB}(15 \% ; P<0.0001)$ and $-1.11 \pm$ 

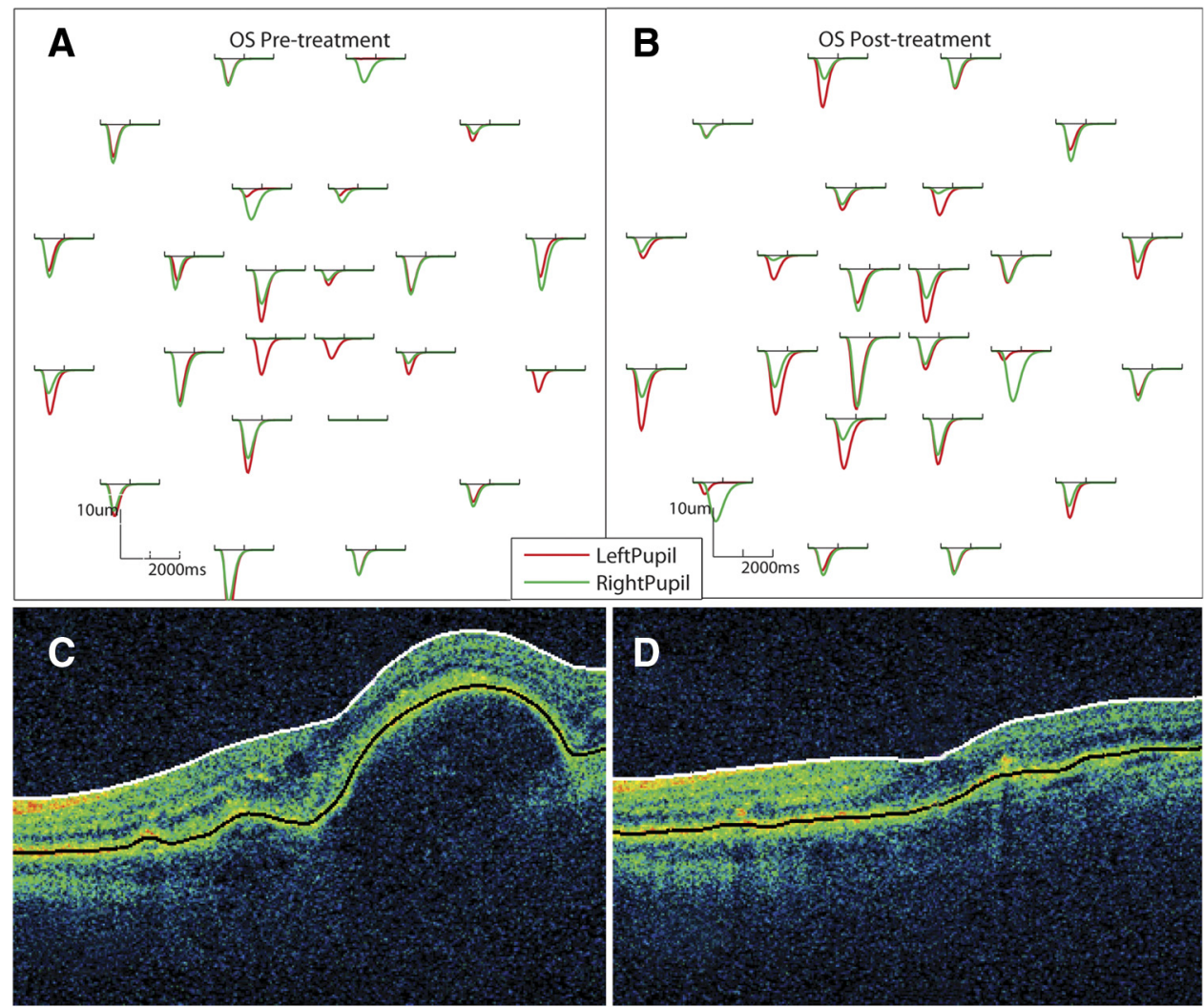

Figure 2. Example of changes in mfPOP and OCT during the course of treatment in subject 417 , who had exudative AMD OS. (A) Mean direct and consensual regional response recordings to the T24 stimulus ensemble at pretreatment. (B) Three months after intravitreal ranibizumab injection. A downward deflection represents a pupil contraction response to the stimuli presented at that location. (C, E) OCT line scan and retinal thickness map of the same eye demonstrated severe retinal edema corresponding to regions of reduced pupillary response amplitudes. (D, F) After 3 months of treatment, recovery from edema temporal to the fovea showed an improvement in afferent contraction amplitude responses in the nasal visual field, with direct and consensual responses mostly overlapping.

$0.24 \mathrm{~dB}(23 \% ; P<0.00001)$ smaller than normals. After treatment, the mean effect of exudative AMD on response amplitudes measured $-0.71 \pm 0.17 \mathrm{~dB}(P<0.0001)$ and $-0.53 \pm$ $0.22 \mathrm{~dB}(P=0.019)$ for stimulus $\mathrm{O} 44$ and T24, respectively. Independent effects on time to peak responses (Table 3) were more severely affected than contraction amplitudes with responses significantly more delayed than normal by $16.22 \pm$ $2.10 \mathrm{~ms}\left(P<5 \times 10^{-14}\right)$ for stimulus $\mathrm{O} 44$ and $18.82 \pm 3.0 \mathrm{~ms}$ $\left(P<5 \times 10^{-10}\right)$ for stimulus T24. After 3 months of treatment, delays due to AMD declined to $15.05 \pm 2.20 \mathrm{~ms}(P<5 \times$ $\left.10^{-11}\right)$ and $7.45 \pm 3.15 \mathrm{~ms}(P<0.05)$ for stimulus $\mathrm{O} 44$ and

TABLE 2. Summary of Independent Effects of Variables on Pupil Contraction Amplitudes Estimated by a Multiple Linear Model

\begin{tabular}{|c|c|c|c|c|c|c|}
\hline \multirow[b]{2}{*}{ Variable } & \multicolumn{3}{|c|}{044} & \multicolumn{3}{|c|}{ T24 } \\
\hline & dB & SE & $\boldsymbol{P}$ & dB & SE & $\boldsymbol{P}$ \\
\hline Reference & 4.91 & 0.30 & $*$ & 3.94 & 0.33 & $*$ \\
\hline Consensual & -0.04 & 0.08 & 0.632 & -0.13 & 0.12 & 0.287 \\
\hline Female & 0.16 & 0.10 & 0.118 & 0.05 & 0.14 & 0.729 \\
\hline AMD before treatment & -0.71 & 0.17 & $*$ & -1.11 & 0.24 & * \\
\hline AMD after treatment & -0.71 & 0.17 & $*$ & -0.53 & 0.22 & 0.019 \\
\hline
\end{tabular}

$$
{ }^{*} P<5 \times 10^{-4} \text {. }
$$

T24, respectively. Overall, time to peak responses in the women were on average between $9.45 \pm 1.92 \mathrm{~ms}(P<5 \times$ $\left.10^{-7}\right)$ to $34.52 \pm 1.34 \mathrm{~ms}\left(P<5 \times 10^{-142}\right)$ quicker than those in the men. The mean consensual responses were not significantly different from direct responses, consistent with previous studies. ${ }^{13,15}$ Stimulus T24 elicited the largest and most significant effect for both contraction amplitudes and delays before treatment in AMD patients; however, this trend was not observed after treatment. A separate analysis identified additional delays to be significantly shorter after 3 months of treatment for both stimuli O44 $\left(P<5 \times 10^{-4}\right)$ and T24 $(P<$

Table 3. Summary of Independent Effects of Variables on Time to Peak Contraction

\begin{tabular}{|c|c|c|c|c|c|c|}
\hline \multirow[b]{2}{*}{ Variable } & \multicolumn{3}{|c|}{044} & \multicolumn{3}{|c|}{ T24 } \\
\hline & ms & SE & $\boldsymbol{P}$ & ms & SE & $\boldsymbol{P}$ \\
\hline Reference & 520 & 4.29 & $*$ & 494 & 4.76 & $*$ \\
\hline Consensual & 2.04 & 1.24 & 0.092 & 2.59 & 1.74 & 0.137 \\
\hline Female & -34.52 & 1.34 & $*$ & -9.45 & 1.92 & * \\
\hline AMD before treatment & 16.22 & 2.10 & $*$ & 18.82 & 3.00 & * \\
\hline AMD after treatment & 15.05 & 2.20 & * & 7.45 & 3.15 & 0.018 \\
\hline
\end{tabular}

$$
{ }^{*} P<5 \times 10^{-6} \text {. }
$$


$\left.5 \times 10^{-9}\right)$. By contrast, there was only minor improvement in mean contraction amplitude deviation at posttreatment for protocol T24 $(P=0.017)$.

Presenting region-by-region amplitude and delay deviations from normal responses (Fig. 3) for stimulus T24 allows for affected locations to be investigated over time. Responses from both eyes were combined so that each visual field location represented a similar nasotemporal region in each eye. The result at the baseline visit pointed to retinal dysfunction in patients with exudative AMD. Figures $3 \mathrm{~A}$ and $3 \mathrm{C}$ indicate that the longest delays occur within the central $3^{\circ}$ of fixation. Figure 3B shows shorter mean additional delays in exudative AMD (shown as darker grays) and a bias toward less delay in the nasal field, evident at 3 months after treatment. To determine whether retinal dysfunction was deteriorating more in the foveal than in the parafoveal area, we analyzed the mean effects at baseline and 3 months using averages across the three concentric rings of the T24 stimulus (Figs. 3C, 3F). Figure $3 \mathrm{C}$ demonstrates additional delay effects reaching a peak in ring 1 corresponding to the foveal region $\left( \pm 3^{\circ}\right.$ retinal eccentricity). On average, additional delay effects at 3 months (Fig. 3C) were significantly smaller for all concentric rings $(P<$ 0.05 , paired $t$-test). Average contraction amplitude deviations were largest for ring 2 (Fig. 3F) at baseline, with seven regions within the parafovea having significantly smaller responses
$(P<0.05)$ than normal (Fig. 3D). An improvement in mean response amplitude deviation was evident at posttreatment follow-up (Figs. 3E, 3F), but reached significance only in ring 2 . Thus the region-wise analysis was more revealing than the ring analysis.

We also examined the predictive capacity of mfPOP by fitting an effect for subjects who demonstrated improved acuity or reduced CRT at 3 months. On average, subjects who displayed a decrease in CRT contracted by an additional $0.54 \pm$ $0.15 \mathrm{~dB}(11.4 \%)$ and were $21.3 \pm 2.6 \mathrm{~ms}$ quicker to stimulus T24 after 3 months (both $P<0.005$ ). When the correlation between mfPOP amplitude and time to peak responses and BCVA were analyzed, no significant correlation was found before and after treatment examinations $(P=0.76, P=0.73$, $P=0.58, P=0.57$, respectively). Figure 4 shows the baseline mean regional amplitude deviation profile of subjects who had reductions in CRT at 3 months for stimulus O44 (Fig. 4A) and T24 (Fig. 4B). The results show a strong consensus between stimulus protocols with small but significantly larger than normal mean responses (lightest regions) in inferior peripheral regions.

The diagnostic power achieved in the mfPOP stimulus protocols for exudative AMD at baseline and posttreatment are shown in Table 4. This also showed the value of a higher resolution analysis of the visual field. AUC values for response
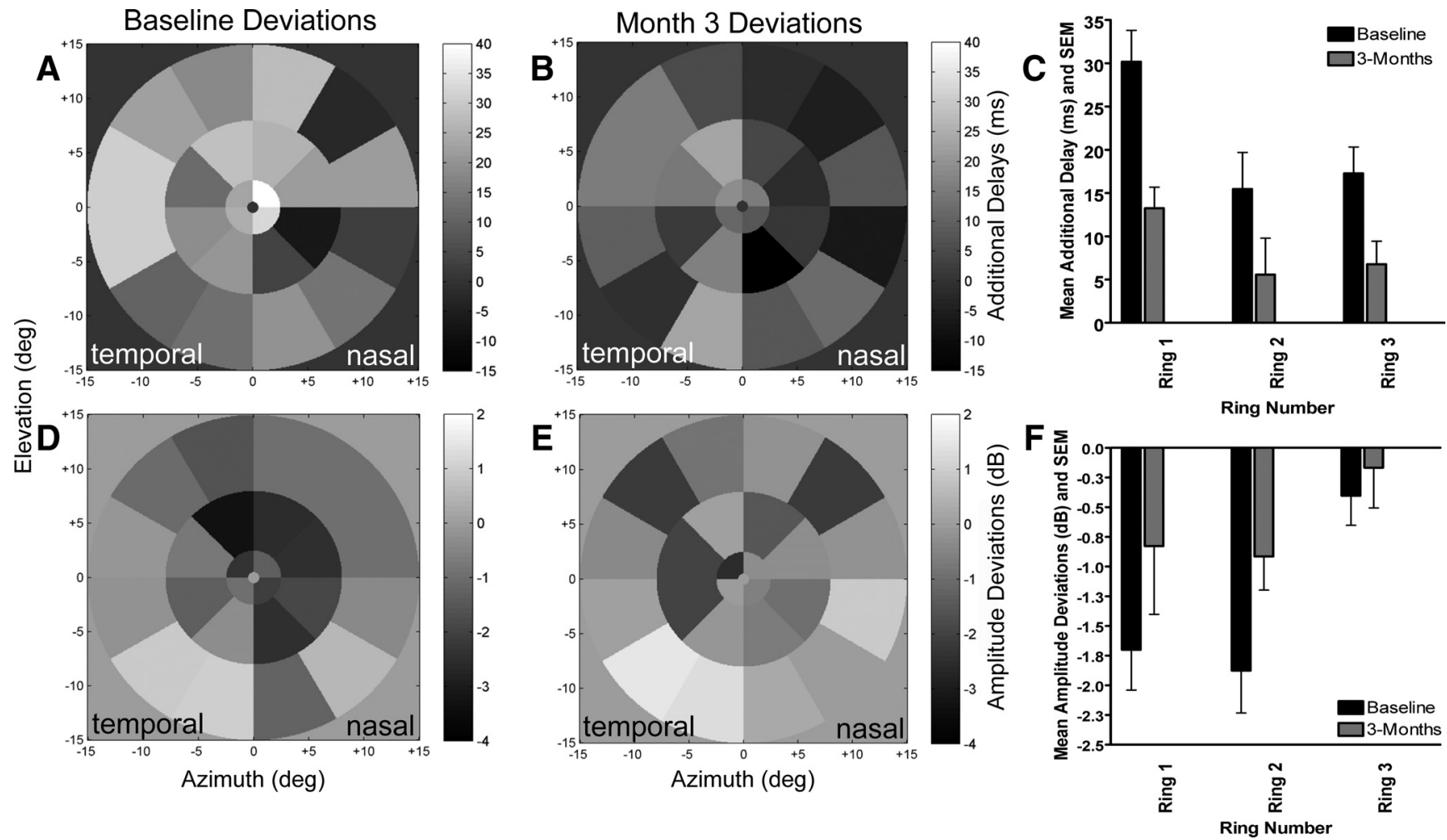

FIGURE 3. Measures of regional mean pupil response deviations from normal, before and 3 months after intravitreal ranibizumab injection. The means are quantified by a multiple regression model where the subject's sex and consensual response were also factors. Contraction amplitudes and delays from both eyes have been combined by reversing the results of the right eye such that each region represents the identical segment of the field for each eye; thus, the temporal field is on the left. The background gray level represents a deviation of 0 . (A) Mean additional delays caused by exudative AMD subjects at baseline, thus longer delays are depicted in lighter gray. The delays of the brightest 10 regions in the field reached statistical significance. (B) Mean additional delays by region 3 months after onset of treatment. No regions were statistically more delayed than normal. (C) Relationship between mean additional delays at baseline and 3 months across increasing ring eccentricity. All rings showed a significant reduction of response delays at 3 months $(P<0.05)$. (D) The mean amplitude deviations for exudative AMD subjects at baseline. The seven darkest regions that fall within the parafovea were significantly reduced in amplitude. (E) Mean amplitude deviations at 3 months after treatment. Contraction amplitude deviations from 3 regions reached significance across the central $15^{\circ}$ of visual field. Six regions from the outer ring appeared to be hypersensitive, but that effect did not reach statistical significance. (F) Relationship between mean amplitude deviations at baseline and 3 months according to rings. A statistically significant change in amplitude deviations was observed only in ring 2 at the second examination. 

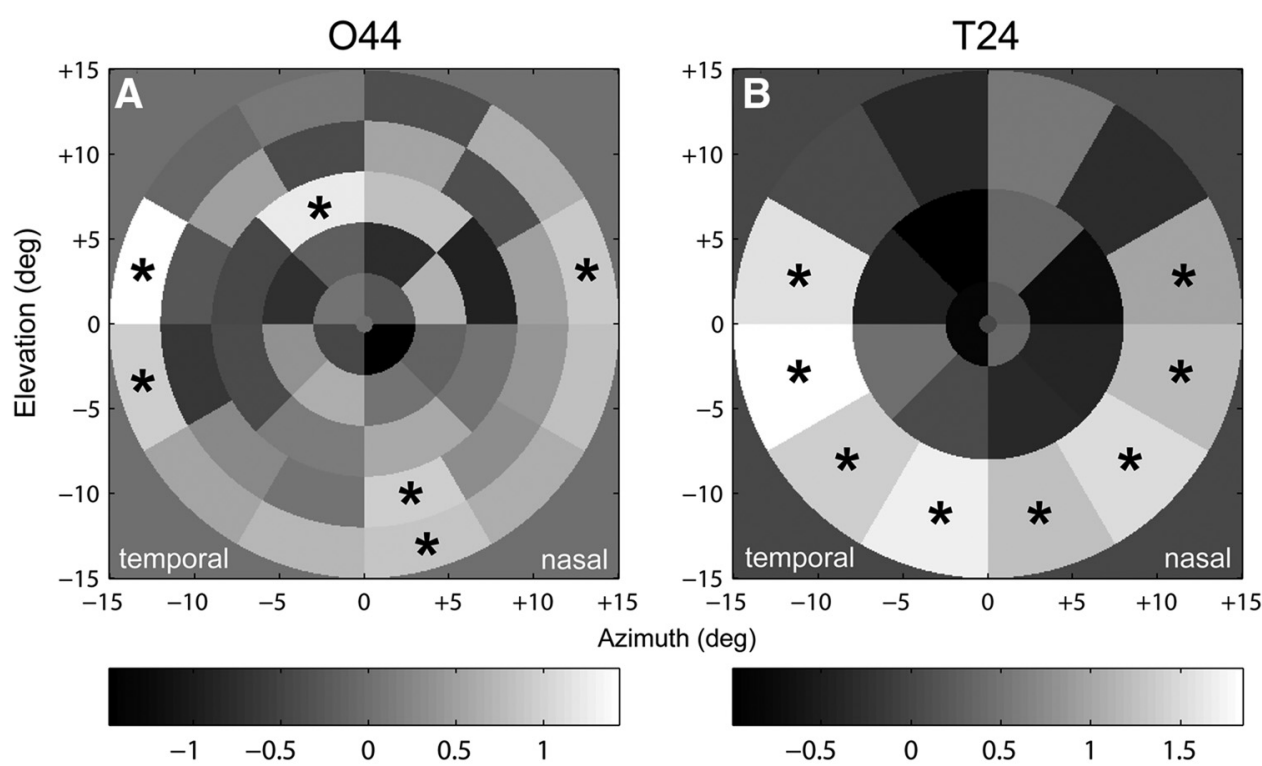

Azimuth (deg)

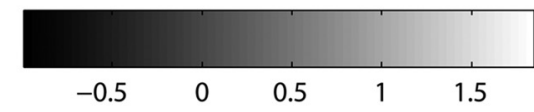

Figure 4. Baseline mean regional contraction amplitude deviations representing subjects who had improvements in CRT after ranibizumab therapy. (A) Stimulus O44 shows a bias toward larger response amplitudes in the inferior hemifield with six regions reaching significance $\left({ }^{*} P<0.05\right)$. (B) Seven regions in the inferior peripheral annulus of stimulus T24 produced significantly larger responses than normal. delays, illustrated that stimulus $\mathrm{O} 44$ achieved the highest diagnostic performance at both baseline $(100 \% \pm 0.0 \%)$ and month $3(100 \% \pm 0.0 \%)$ examinations. Evaluating the mean percent AUC and SE obtained for the worst-deviating (N-worst) 2, 10, and all regions of the visual field suggests that restricting the analysis to the 1 or 2 worst-performing regions improves diagnostic performance and confirms our expectation of the presence of localized severe retinal dysfunction in exudative AMD. We also assessed diagnostic accuracy using constriction amplitudes and found that it did not perform as well at baseline $(96.9 \% \pm 3.1 \%)$ but was consistent with time to peak deviations after treatment $(96.8 \% \pm 4.4 \%)$.

\section{Discussion}

This study has investigated changes in retinal function as measured by mfPOP in eyes with exudative AMD before and after intravitreal ranibizumab therapy for $\mathrm{CNV}$. The method identified functional impairment in contraction amplitudes and time to peak responses within the central $15^{\circ}$ of fixation. A significant reduction in CRT $(P<0.001)$ and additional delays for stimulus protocol O44 $\left(P<5 \times 10^{-4}\right)$ and T24 $\left(P<5 \times 10^{-9}\right)$ were observed at 3 months after treatment. In contrast with earlier studies, ${ }^{6-8}$ we did not observe a significant mean im- provement in BCVA after treatment; however, baseline vision was better in this series than in the pivotal trials.

The results of our study suggest that intravitreal ranibizumab injections had larger effects on time to peak responses than on contraction amplitudes. At baseline (Fig. 3A), we identified 10 visual field regions that were significantly delayed $(P<0.05)$ from normal, whereas at the 3-month follow-up, there were no regions significantly delayed, although we did observe a bias toward shorter delays in the nasal visual field (Fig. 3B). Contraction amplitude deviations were less informative than response delays, with only a marginally significant reduction in the mean effect of exudative AMD after 3 months of treatment (Table 2) for stimulus T24. Mean regional amplitude deviations from normative data peaked in the parafoveal region (ring 2) of the visual field (Figs. 3D, 3F) confirming the results of our first published mfPOP study of exudative AMD ${ }^{23}$ which is in accordance with histopathological findings of the initial site of photoreceptor loss (0.5-2 $\mathrm{mm}$ from the fovea) in AMD. ${ }^{24}$ What was striking was the three regions demonstrating hypersensitivity in contraction amplitudes (Fig. 3E) in the outer ring of exudative AMD subjects after ranibizumab therapy. Previous studies from our laboratory have found positive mean effects for peripheral contraction amplitude deviations in diabetic retinopathy ${ }^{14}$ and exudative AMD. ${ }^{23}$ Of particular in-

TABLE 4. ROC Percentage AUC of Time to Peak Contraction (Delay) Presented as the Values at Baseline and after Treatment

\begin{tabular}{|c|c|c|c|c|c|c|}
\hline & \multicolumn{3}{|c|}{044} & \multicolumn{3}{|c|}{ T24 } \\
\hline & AUC $(n=2)$ & $\operatorname{AUC}(n=10)$ & AUC $(n=$ All $)$ & $\operatorname{AUC}(n=2)$ & $\operatorname{AUC}(n=10)$ & AUC $(n=$ All $)$ \\
\hline Baseline & $100 \pm 0.0$ & $95.0 \pm 3.9$ & $94.2 \pm 4.0$ & $95.8 \pm 4.1$ & $85.8 \pm 5.5$ & $82.3 \pm 6.4$ \\
\hline Month 3 & $100 \pm 0.0$ & $98.8 \pm 1.1$ & $96.8 \pm 2.2$ & $91.1 \pm 6.0$ & $83.8 \pm 7.0$ & $79.3 \pm 7.9$ \\
\hline
\end{tabular}

ROC AUC data (mean $\% \pm$ SE) of 20 eyes with exudative AMD at baseline and 3 months after treatment for stimulus $\mathrm{O} 44$ and T24 are presented. The next three columns to the right present AUC and SE results for the 2, 10, and all worst deviations from normal (N-worst). All deviations are equivalent to using the mean defect in perimetry. Diagnostic performance was assessed on between-eye deviations from normative data for time to peak contraction (delay). This result was determined by measuring the differences in deviations at the same VF region between eyes. The diagnostic performance of stimulus $\mathrm{O} 44$ was greater than T24 for all N-worst deviations considered. 
terest are the baseline response deviations of subjects who had significant reductions in CRT over time (Figs. 4A, 4B). The results, if confirmed in a larger group of subjects, suggest that increased response amplitudes in the inferior peripheral regions may be a unique functional marker for predicting CRT outcomes after ranibizumab therapy. We propose that in an attempt to improve central retinal sensitivity a global mechanism may be attempting to increase response gain generally to compensate for disease, which acts to boost responses from healthy peripheral regions. Detailed investigation into changes in retinal sensitivity measured by $\mathrm{mfPOP}$ and changes in retinal structure are needed, to confirm that hypothesis.

The observed disparity between response delays and amplitudes may reflect disruptions to interneurons in diseases of the outer retina. ${ }^{25-27}$ Temporal responses may be affected to a greater extent than other parameters of the pupillary signal. This imbalance has been reported in $\mathrm{mfERG},{ }^{28}$ and multifocal pupillography studies in patients with retinitis pigmentosa.? Another hypothesis is that morphologic changes in the RPE/ Bruch's membrane complex, such as retinal pigment epithelial abnormalities and drusen, both known to significantly reduce macular sensitivity, ${ }^{29}$ may contribute to a timing delay. A limitation of our study is the difficulty of assessing the degree of overlap between changes in retinal morphology and visual dysfunction.

Superimposition of the regional mfPOP data on the OCT and fundus images must be examined to determine the correlation between structure and function.

Kiss et al. ${ }^{6}$ investigated the effects of ranibizumab therapy on central retinal sensitivity with microperimetry in patients with CNV secondary to exudative AMD. They found that mean retinal sensitivity improved from baseline, for the foveal center $\left(0^{\circ}\right)$ and paracentral test locations $\left(3.5^{\circ}\right.$ retinal eccentricity), after 3 months of ranibizumab therapy. At the extrafoveal regions $\left(7.9^{\circ}\right)$ a decrease in sensitivity was observed after ranibizumab therapy. A separate study by the same author investigating combination verteporfin photodynamic therapy (PDT) and ranibizumab found no significant changes in mean retinal sensitivity outside the regions treated with PDT. ${ }^{8}$ They suggested that ranibizumab had no measurable effect on regions of healthy retina. In agreement with Kiss et al., ${ }^{6,8}$ we found improvements in the overall mean effect of exudative AMD on contraction amplitudes and time to peak at 3 months, with reductions in amplitude deviations less pronounced beyond $\pm 7.7^{\circ}$ retinal eccentricity (Fig. 3F). In addition, differences may exist in the vulnerability of ganglion cell type and organization between the visual and pupil pathways in outer retinal disease, manifesting distinct patterns of dysfunction for mfPOP and microperimtrey. An apparent advantage of mfPOP over other objective perimeters (mfVEP and mfERG) lies in its noninvasive nature, minimal setup, and brief testing time.

Stimulus protocols with high-resolution layouts (e.g., 44 regions/field) produced smaller effects for exudative AMD on contraction amplitudes than low-resolution stimuli (T24). By contrast the relatively high resolution of contraction delays for the 044 stimuli was definitely more diagnostically useful that the lower resolution T24 ensemble (Table 4). We have attempted higher resolution stimuli having 24 or 44 regions within the central $20^{\circ}$, but these so far have provided poor signal to noise ratios. ${ }^{15}$ At the same time, large blurry stimuli are tolerant of misrefraction and have been suggested to reduce test-retest variability. ${ }^{19}$ AUC values were a bit lower after treatment (Table 4), seemingly quantifying the improvement in retinal function (e.g., Fig. 2)

In summary, we describe the first investigation of macular dysfunction by mfPOP after ranibizumab therapy. Although previous studies reported effects on retinal sensitivity, we observed clinically meaningful changes in the temporal com- ponent of the regional pupillary response and demonstrated the diagnostic potential of mfPOP. The presence of increased response amplitudes in the inferior peripheral regions was the best predictor of improvement in CRT after treatment. Prognostic markers identifying potential recovery of function will gain significance for optimizing patient selection as new treatment options for exudative AMD develop. In addition, significant improvement in regional temporal responses after treatment may help guide retreatment strategies. A combined mfPOP and CRT approach may allow us to monitor patients more precisely and initiate treatment earlier. Longitudinal prospective studies with larger patient numbers are needed, to determine whether improvements in mfPOP responses are maintained during ranibizumab treatment.

\section{References}

1. Friedman DS, O'Colmain BJ, Munoz B, et al. Prevalence of agerelated macular degeneration in the United States. Arch Opbthalmol. 2004;122:564-572.

2. Kvanta A, Algvere PV, Berglin L, Seregard S. Subfoveal fibrovascular membranes in age-related macular degeneration express vascular endothelial growth factor. Invest Opbthalmol Vis Sci. 1996;37: 1929-1934

3. Lopez PF, Sippy BD, Lambert HM, Thach AB, Hinton DR. Transdifferentiated retinal pigment epithelial cells are immunoreactive for vascular endothelial growth factor in surgically excised age-related macular degeneration-related choroidal neovascular membranes. Invest Ophthalmol Vis Sci. 1996;37:855-868.

4. Brown DM, Kaiser PK, Michels M, et al. Ranibizumab versus verteporfin for neovascular age-related macular degeneration. $N$ Engl J Med. 2006;355:1432-1444.

5. Rosenfeld PJ, Brown DM, Heier JS, et al. Ranibizumab for neovascular age-related macular degeneration. $N$ Engl J Med. 2006;355: 1419-1431.

6. Kiss CG, Geitzenauer W, Simader C, Gregori G, Schmidt-Erfurth U. Evaluation of ranibizumab-induced changes in high-resolution optical coherence tomographic retinal morphology and their impact on visual function. Invest Ophthalmol Vis Sci. 2009;50:23762383.

7. Frennesson C, Nilsson UL, Peebo BB, Nilsson SE. Significant improvements in near vision, reading speed, central visual field and related quality of life after ranibizumab treatment of wet agerelated macular degeneration. Acta Opbthalmol. 88:420-425.

8. Kiss CG, Simader C, Michels S, Schmidt-Erfurth U. Combination of verteporfin photodynamic therapy and ranibizumab: effects on retinal anatomy, choroidal perfusion and visual function in the protect study. Br J Ophthalmol. 2008;92:1620-1627.

9. Tan L, Kondo M, Sato M, Kondo N, Miyake Y. Multifocal pupillary light response fields in normal subjects and patients with visual field defects. Vision Res. 2001;41:1073-1084.

10. Wilhelm H, Neitzel J, Wilhelm B, et al. Pupil perimetry using M-sequence stimulation technique. Invest Opbthalmol Vis Sci. 2000;41:1229-1238.

11. James AC, Ruseckaite R, Maddess T. Effect of temporal sparseness and dichoptic presentation on multifocal visual evoked potentials. Vis Neurosci. 2005;22:45-54

12. Maddess T, James AC, Bowman EA. Contrast response of temporally sparse dichoptic multifocal visual evoked potentials. Vis Neurosci. 2005;22:153-162.

13. Carle CF, James AC, Kolic M, Loh YW, Maddess T. High-resolution multifocal pupillographic objective perimetry in glaucoma. Invest Opbthalmol Vis Sci. 2011;52:604-610.

14. Bell A, James AC, Kolic M, Essex RW, Maddess T. Dichoptic multifocal pupillography reveals afferent visual field defects in early type 2 diabetes. Invest Ophthalmol Vis Sci. 2010;51:602608.

15. Sabeti F, James AC, Maddess T. Spatial and temporal stimulus variants for multifocal pupillography of the central visual field. Vision Res. 2011;51:303-310.

16. Brown DM, Michels M, Kaiser PK, Heier JS, Sy JP, Ianchulev T. Ranibizumab versus verteporfin photodynamic therapy for neovas- 
cular age-related macular degeneration: two-year results of the ANCHOR study. Ophthalmology. 2009;116:57-65 e55.

17. Maddess T, Bedford SM, Goh XL, James AC. Multifocal pupillographic visual field testing in glaucoma. Clin Exp Ophthalmol. 2009;37:678-686.

18. Maddess T, Ho YL, Wong SS, et al. Multifocal pupillographic perimetry with white and colored stimuli. J Glaucoma. 2011;20: $336-343$.

19. Maddess T. The influence of sampling errors on test-retest variability in perimetry. Invest Ophthalmol Vis Sci. 2011;52:1014-1022.

20. James AC. The pattern-pulse multifocal visual evoked potential. Invest Ophthalmol Vis Sci. 2003;44:879-890.

21. James AC, Kolic M, Bedford SM, Maddess T. Stimulus parameters for multifocal pupillographic objective perimetry. J Glaucoma. Published online May 26, 2011.

22. Hood DC, Zhang X, Greenstein VC, et al. An interocular comparison of the multifocal VEP: a possible technique for detecting local damage to the optic nerve. Invest Ophthalmol Vis Sci. 2000;41: $1580-1587$.
23. Sabeti F, Maddess T, Essex RW, James AC. Multifocal pupillographic assessment of age-related macular degeneration. Optom Vis Sci. 2011;88:1477-1485.

24. Curcio CA, Medeiros NE, Millican CL. Photoreceptor loss in agerelated macular degeneration. Invest Opbthalmol Vis Sci. 1996; 37:1236-1249.

25. Fariss RN, Li ZY, Milam AH. Abnormalities in rod photoreceptors amacrine cells, and horizontal cells in human retinas with retinitis pigmentosa. Am J Opbthalmol. 2000;129:215-223.

26. Fletcher EL, Kalloniatis M. Neurochemical architecture of the normal and degenerating rat retina. J Comp Neurol. 1996;376:343-360.

27. Li ZY, Kljavin IJ, Milam AH. Rod photoreceptor neurite sprouting in retinitis pigmentosa. J Neurosci. 1995;15:5429-5438.

28. Hood DC, Holopigian K, Greenstein V, et al. Assessment of local retinal function in patients with retinitis pigmentosa using the multi-focal ERG technique. Vision Res. 1998;38:163-179.

29. Midena E, Vujosevic S, Convento E, Manfre A, Cavarzeran F, Pilotto E. Microperimetry and fundus autofluorescence in patients with early age-related macular degeneration. Br J Opbthalmol. 2007;91: $1499-1503$. 